07

\title{
Вязкоупругость и механизмы пластичности дентина зубов человека
}

\author{
(C) И.Н. Бородин ${ }^{1-3}$, S. Seyedkavoosi ${ }^{4}$, Д.В. Зайцев ${ }^{1}$, B. Drach ${ }^{4}$, \\ К.Н. Микаелян ${ }^{3}$, П.Е. Панфрилов ${ }^{1}$, М.Ю. Гуткин ${ }^{3,5,6, \text { I , I. Sevostianov }}{ }^{4}$ \\ ${ }^{1}$ Уральский фредеральный университет, Институт естественных наук, \\ Екатеринбург, Россия \\ 2 Челябинский государственный университет, \\ Челябинск, Россия \\ ${ }^{3}$ Институт проблем машиноведения РАН, \\ Санкт-Петербург, Россия \\ ${ }^{4}$ Department of Mechanical and Aerospace Engineering, New Mexico State University, \\ Las Cruces, NM, USA \\ ${ }^{5}$ Санкт-Петербургский политехнический университет Петра Великого, \\ Санкт-Петербург, Россия \\ ${ }^{6}$ Университет ИТМО, \\ Санкт-Петербург, Россия \\ ๑ E-mail: m.y.gutkin@gmail.com \\ (Поступила в Редакцию 14 июня 2017 г.)
}

Рассмотрены теоретические модели вязкоупругого поведения и механизмов пластической деформации дентина зубов человека. С помощью теории линейной вязкоупругости, в которой ядра ползучести и релаксации имеют вид дробно-экспоненциальных функций, найдены численные значения мгновенного и долговременного модулей Юнга, а также других характеристик вязкоупругости дентина при одноосном сжатии. В качестве механизмов пластической деформации дентина предложены взаимное проскальзывание коллагеновых волокон в месте контакта их боковых поверхностей, отрыв этих волокон друг от друга и необратимое растяжение отдельных коллагеновых волокон. Показано, что для срабатывания второго механизма требуется меньшее напряжение, чем для срабатывания двух других. Исследованы модели соответствующих этим механизмам пластических зон в вершине трещины простого отрыва. Показано, что с ростом приложенного напряжения размер пластической зоны может вырасти от нескольких сотен нанометров до сотни микрометров.

Работа выполнена при поддержке Российского научного фонда (грант РНФ № 15-19-10007).

DOI: 10.21883/FTT.2018.01.45298.188

\section{1. Введение}

Дентин - это твердая ткань, которая составляет основу зубов человека и животных [1-8]. В области коронки дентин покрыт эмалью, в области корня - цементом. По массе (объему) дентин примерно на 70\% (50\%) состоит из неорганических веществ, на 20\% (25\%) из органических и на $10 \%$ (25\%) из воды. По структуре его можно отнести к развитым природным иерархическим композитам с тремя основными масштабными уровнями (рис. 1).

Первый уровень представлен отдельными органическими волокнами коллагена 1-го типа диаметром порядка $100 \mathrm{~nm}$ и неорганическими нанокристаллами гидроксиапатита кальция размером 20-50 nm, которые заполняют пространство между коллагеновыми волокнами (КВ). КВ - это пучки фибрилл, которые представляют собой полукристаллические структуры, образующие основную структурную единицу коллагена. Отдельные фибриллы имеют вид тонких удлиненных структур диаметром от 20 до $90 \mathrm{~nm}$, которые могут достигать нескольких микрометров в длину. Они обладают характерной периодичностью в 64-67 nm, вызванной пере- крывающимся расположением спиралевидных молекул тропоколлагена, которые соединены между собой некоторым непостоянным количеством ковалентных связей.

На втором масштабном уровне КВ образуют сеточную структуру, которая заполняет пространство между дентинными канальцами (ДК) - длинными однонаправленными полостями диаметром $3-5 \mu \mathrm{m}$, отстоящими друг от друга на расстояния порядка $10 \mu \mathrm{m}$, по которым циркулирует дентинная жидкость. КВ ориентированы преимущественно перпендикулярно ДК.

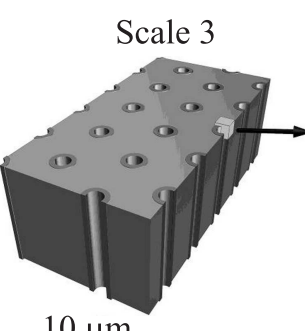

$10 \mu \mathrm{m}$

\section{Scale 2}

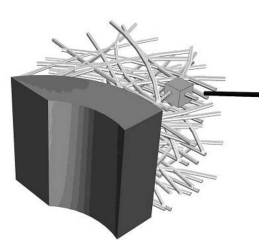

$\underline{1 \mu \mathrm{m}}$
Scale 1

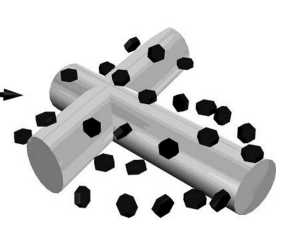

$100 \mathrm{~nm}$
Рис. 1. Схематичное представление масштабных уровней дентина. 
Третий масштабный уровень формируют сами ДК, окруженные высокоминерализованной оболочкой перитубулярным дентином, и промежуточный, менее минерализованный интертубулярный (межтрубочный) дентин.

Композитная структура дентина позволяет ему сочетать упругость и прочность, достаточные для того, чтобы зуб мог выдерживать значительные механические нагрузки при откусывании и пережевывании пищи и в то же время оставаться живым органом, который развивается в течение всей жизни своего обладателя. Механические свойства дентина начали изучать еще в конце XIX века, и до сих пор они привлекают большое внимание исследователей (см., например, относительно недавние обзоры [9-11]).

По-видимому, первое систематическое экспериментальное исследование механических свойств дентина при сжатии было выполнено американским зубным врачом д-ром Дж.В. Блэком, который опубликовал свои результаты в серии статей [12] (полный текст доступен в Интернете на сайте [13]). В частности, он показал, что расположение и ориентация ДК существенно не влияют на свойства дентина. Этот результат был позже подтвержден в работах $[14,15]$. Авторы [15] также показали, что свойства дентина трансверсально-изотропны.

В работе [16] для измерения механических свойств деминерализованного человеческого дентина использовался атомно-силовой микроскоп. Образцы дентина исследовались в трех состояниях: в воде, на воздухе после высушивания и в воде после регидратации. Авторы пришли к заключению, что КВ практически не влияют на жесткость дентина, однако дают существенный вклад в его прочность и вязкость разрушения.

Упругое поведение дентина изучалось также при испытаниях на растяжение малых образцов, вырезанных вдоль и поперек ДК [17]. Было показано, что упругие свойства дентина почти не зависят от ориентации ДК. Измеренная величина эффективного модуля Юнга составила $29.5 \mathrm{GPa}$, коэффициента Пуассона - 0.44 .

Подробный критический обзор результатов экспериментов по определению механических свойств человеческого дентина, выполненных во второй половине XX века, приведен в работе [10]. Здесь подытожены результаты измерения показателей упругости, твердости, прочности, вязкости разрушения и усталости дентина. Рассмотрены также характеристики его вязкоупругого поведения. В то же время, целый ряд работ, посвященных изучению вязкоупругости, либо остались за кадром, либо появились позже. Перечислим кратко основные результаты, полученные в этом направлении в последние годы.

Экспериментальное изучение ползучести, релаксации напряжений и скоростной зависимости деформации показало линейную вязкоупругость корневого человеческого дентина при сжатии [18]. В работе [19] исследовались кривые релаксации напряжений при растяжении дентина, и авторы пришли к противоположному выводу о его нелинейной вязкоупругости. Еще раньше при испытаниях на релаксацию напряжений [20] было показано, что модуль релаксации дентина линейно зависит от логарифма времени в течение примерно четырех десятилетий, и что распределение времен релаксации для выбранной ориентации образцов в значительной степени постоянно в течение этого периода времени. Недавнее систематическое экспериментальное исследование in vitro [21-23] деформационного поведения дентина при сжатии с учетом влияния размера и формы образцов, а также скорости нагружения, показало, что его механические свойства существенно зависят от этих параметров, что, в частности, объясняется авторами пластичностью органической составляющей дентина, представленной КВ, и пористостью межтрубочкового дентина. Эти эксперименты также подтвердили представление о дентине как о вязкоупругом материале, способном выдерживать большие упругие и значительные необратимые деформации, а также эффективно сопротивляться развитию трещин [24]. Экспериментальному определению характеристик вязкоупругости человеческого дентина при одноосном сжатии посвящена работа [25], в которой продемонстрирована линейная вязкоупругость дентина при „клинических“ уровнях сжимающего напряжения с максимальной деформацией порядка 1\%. Отметим также исследования по измерению модуля Юнга, твердости и других механических характеристик дентина, включая характеристики ползучести, методом наноиндентирования (см. работу [26] и приведенные в ней ссылки).

Теоретическому описанию вязкоупругого поведения дентина посвящены работы [27-29]. В частности, в работе [27] предложена модель, описывающая эксперименты [17]. Используя определяющее соотношение вязкоупругости для анизотропного композитного материала с цилиндрическими порами, авторы [28] разработали модель, позволяющую предсказывать деформацию при ползучести, релаксацию напряжений и мгновенную упругость дентина без трещин. Модель, нацеленная на количественное описание характеристик упругости и вязкоупругости деминерализованного дентина при испытаниях на ползучесть в процессе наноиндентирования, предложена в работе [29]. Заметим, что перечисленные модели анализировались довольно формально, без физически обоснованного выбора конкретных значений параметров модели.

Механическое поведение дентина на стадии перехода к разрушению систематически исследовалось в серии работ [30-32], в которых особое внимании уделялось росту трещин, определению коэффициента интенсивности напряжений [31] и влиянию ориентации ДК и КВ на рост трещины [31]. Авторы также предложили общую схему разрушения дентина, в которой ключевую роль играют ДК $[31,32]$. Вместе с тем, приведенные в [30-32] микрофотографии роста трещин показывают, что они растут в коллагеновой матрице независимо от ДК. Аналогичный вывод следует и из экспериментов [33,34]. Интересно, что перед вершиной трещины авторы $[33,34]$ 
обнаружили пластическую зону, предшествующую разрушению образца. Они также наблюдали образование рядом с основной трещиной нескольких сателлитных трещин, что характерно для разрушения пластичного материала. С другой стороны, известно [5], что при сжатии пластическая деформация образцов дентина может достигать $26 \%$.

Таким образом, несмотря на более чем 120-летнюю историю изучения механического поведения дентина, до сих пор не сложилось единого взгляда на физические механизмы его необратимой (пластической) деформации и разрушения. В настоящей статье предлагаются теоретические модели, разработанные на основе результатов экспериментальных исследований ползучести дентина при одноосном сжатии и наблюдений его разрушения с помощью просвечивающей электронной микроскопии. Первая из изложенных ниже моделей описывает эффективные вязкоупругие свойства дентина при одноосном нагружении в рамках теории линейной вязкоупругости Работнова с использованием дробно-экспоненциальных функций в качестве ядер ползучести и релаксации. Вторая - микромеханизмы необратимой деформации в пластической зоне у вершины трещины простого отрыва.

\section{2. Эфффективные вязкоупругие свойства дентина}

В основу предлагаемой модели положена теория линейной вязкоупругости Работнова [35], в которой в качестве ядра интеграла, описывающего эффект последействия, используются дробно-экспоненциальные функции. В самом общем случае определяющее уравнение линейной вязкоупругости записывается в виде

$$
\varepsilon_{i j}(x, t)=S_{i j k l} \sigma_{k l}(x, t)+\int_{0}^{t} K_{i j k l}(t-\tau) \sigma_{k l}(x, \tau) d \tau,
$$

где $\varepsilon_{i j}-$ тензор деформации, $\sigma_{k l}-$ тензор напряжений, $S_{i j k l}-$ тензор мгновенной упругой податливости 4-го ранга, $K_{i j k l}(t)$ - зависящий от времени $t$ тензор 4-го ранга (ядро ползучести), которое удовлетворяет условию угасания памяти $K_{i j k l}(t \rightarrow \infty) \rightarrow 0$.

С помощью интегрального преобразования Лапласа равенство (1) преобразуется к виду $\bar{\varepsilon}_{i j}(p)=$ $=\bar{S}_{i j k l}(p) \bar{\sigma}_{k l}(p)$, так что решение граничной задачи теории вязкоупругости можно получить из решения соответствующей граничной задачи теории упругости с помощью обратного преобразования Лапласа. В этом случае основные трудности связаны с переходом от изображений к оригиналам, поскольку только самые простые ядра в (1) допускают аналитическое обращение. Однако эти простые ядра не подходят для большинства реальных материалов. Решением проблемы может стать применение в качестве ядер дробно-экспоненциальных функций [35-37] вида

$$
\begin{gathered}
\ni_{\alpha}(\beta, t-\tau)=(t-\tau)^{\alpha} \sum_{n=0}^{\infty} \frac{\beta^{n}(t-\tau)^{n(1+\alpha)}}{\Gamma[(n+1)(1+\alpha)]}, \\
-1<\alpha<0
\end{gathered}
$$

которые допускают аналитическое обращение при преобразовании Лапласа и при этом остаются достаточно общими, чтобы можно было добиться соответствия с экспериментальными данными. Для выполнения условия угасания памяти достаточно потребовать, чтобы выполнялись неравенства $\beta<0$ и $-1<\alpha \leq 0$.

Используя обычное обозначение через $K^{*}$ интегрального оператора типа Вольтерра с ядром $K(t-\tau)$, зависящим от разности аргументов, и понимая под умножением оператора на некоторую функцию вычисление интеграла (если он существует)

$$
K^{*} \cdot f=\int_{-\infty}^{t} K(t-\tau) f(\tau) d \tau,
$$

можно записать результат действия оператора с ядром (2) на постоянную $c$ как

$$
Э_{\alpha}^{*}(\beta) \cdot c=\frac{c}{\beta}\left[M_{1+\alpha}\left(\beta t^{1+\alpha}\right)-1\right],
$$

где $M_{\lambda}(z)-$ функция Миттаг-Лефлера:

$$
M_{\lambda}(z)=\sum_{n=0}^{\infty} \frac{z^{n}}{\Gamma(n \lambda+1)},
$$

которая монотонно убывает от 1 до 0 , так что

$$
\lim _{t \rightarrow \infty}\left[\Im_{\alpha}^{*}(\beta, t) \cdot 1\right]=-1 / \beta .
$$

В случае одноосного растяжения или сжатия, реализованном в наших экспериментах, выражение (1) преобразуется к виду: $\varepsilon(x, t)=\left(E^{*}\right)^{-1} \sigma$, где оператор ползучести $\left(E^{*}\right)^{-1}$ является обратным к оператору релаксации $E^{*}$. Используя ядро (2), можно переписать эти операторы в виде

$$
\begin{aligned}
& \left(E^{*}\right)[\varepsilon(x, t)]=E_{0}\left(\varepsilon(x, t)+\lambda \int_{0}^{t} \ni_{\alpha}\left(\beta, t-t^{\prime}\right) \varepsilon\left(x, t^{\prime}\right) d t^{\prime}\right), \\
& \left(E^{*}\right)^{-1}[\sigma(x, t)]=\frac{1}{E_{0}} \\
& \quad \times\left(\sigma(x, t)-\lambda \int_{0}^{t} \ni_{\alpha}\left(\beta-\lambda, t-t^{\prime}\right) \sigma\left(x, t^{\prime}\right) d t^{\prime}\right),
\end{aligned}
$$

где $E_{0}-$ мгновенный модуль Юнга в направлении нагружения. Эта формула проясняет, в частности, физический смысл параметра $\beta$ - это величина, обратная времени релаксации $\tau$ в степени $1+\alpha$, взятая со знаком 
„минус“": $\beta=-1 / \tau^{1+\alpha}$. Из выражений (6) следует также, что $\lambda=\beta\left(1-\varepsilon_{\max }\right)=\beta\left(E_{0}-E_{\infty}\right) / E_{0}$, где $E_{\infty}-$ модуль Юнга при $t \rightarrow \infty$, а $\varepsilon_{\max }$ - максимальная деформация в направлении нагружения.

Таким образом, вязкоупругое поведение материала при одноосном нагружении описывается четырьмя параметрами: $E_{0}, \alpha, \beta$ (или $\tau$ ) и $\lambda$ (или $E_{\infty}$ ). Поскольку при ползучести и релаксации модуль Юнга с течением времени снижается $\left(E_{0} \geq E_{\infty}\right)$, справедливо неравенство $\beta<\lambda<0$.

Преобразование Лапласа от ядра (2) дает

$$
\mathscr{L}\left[\Im_{\alpha}^{*}(\beta, t)\right] \equiv \int_{0}^{\infty} \Im_{\alpha}^{*}(\beta, t) e^{-t p} d t=\left(p^{1-\alpha}+\beta\right)^{-1} .
$$

Это означает, что, если решение упругой задачи удастся представить в виде рациональной функции переменной $x=p^{1+\alpha}$, то ее образ Лапласа можно будет получить в явном аналитическом виде.

Используя результаты испытаний образцов коронкового дентина на одноосное сжатие в режиме ползучести, из сравнения теоретических и экспериментальных кривых ползучести (рис. 2,a) можно определить значения мгновенного $\left(E_{0}\right)$ и долговременного $\left(E_{\infty}\right)$ модулей упругости: $E_{0}=5.13 \mathrm{GPa}, E_{\infty}=1.83 \mathrm{GPa}$ [38]. При этом полученные из такого сравнения значения параметров вязкоупругости отличались для разных величин сжимающего напряжения $\sigma_{1}$ и $\sigma_{2}$

$$
\begin{aligned}
& \sigma_{1}=300 \mathrm{MPa} \rightarrow \alpha_{1}=-0.8231, \\
& \beta_{1}=-1.5076, \quad \lambda_{1}=-0.9705 ; \\
& \sigma_{2}=450 \mathrm{MPa} \rightarrow \alpha_{2}=-0.8231, \\
& \beta_{2}=-1.0692, \quad \lambda_{2}=-0.6884 .
\end{aligned}
$$

Аналогичная процедура проводилась с экспериментальными результатами работы [18], в которой испытания проводились на образцах корневого дентина при четырех разных значениях сжимающего напряжения (рис. $2, b)$. В этом случае получили $E_{0}=12.18 \mathrm{GPa}$, $E_{\infty}=4.10 \mathrm{GPa}$ и следующий набор параметров:

$$
\begin{gathered}
\sigma_{1}=12.73 \mathrm{MPa} \rightarrow \alpha_{1}=-0.9131, \\
\beta_{1}=-1.1260, \lambda_{1}=-0.7473, \\
\sigma_{2}=38.20 \mathrm{MPa} \rightarrow \alpha_{2}=-0.9131, \\
\beta_{2}=-0.3810, \lambda_{2}=-0.2528, \\
\sigma_{3}=63.66 \mathrm{MPa} \rightarrow \alpha_{3}=-0.9131, \\
\beta_{3}=-0.2159, \lambda_{3}=-0.1433, \\
\sigma_{4}=89.13 \mathrm{MPa} \rightarrow \alpha_{4}=-0.9131, \\
\beta_{4}=-0.1709, \lambda_{4}=-0.1134 .
\end{gathered}
$$

Как и ожидалось, долговременный модуль Юнга $\left(E_{\infty}\right)$ оказался существенно меньше мгновенного $\left(E_{0}\right)$. Для
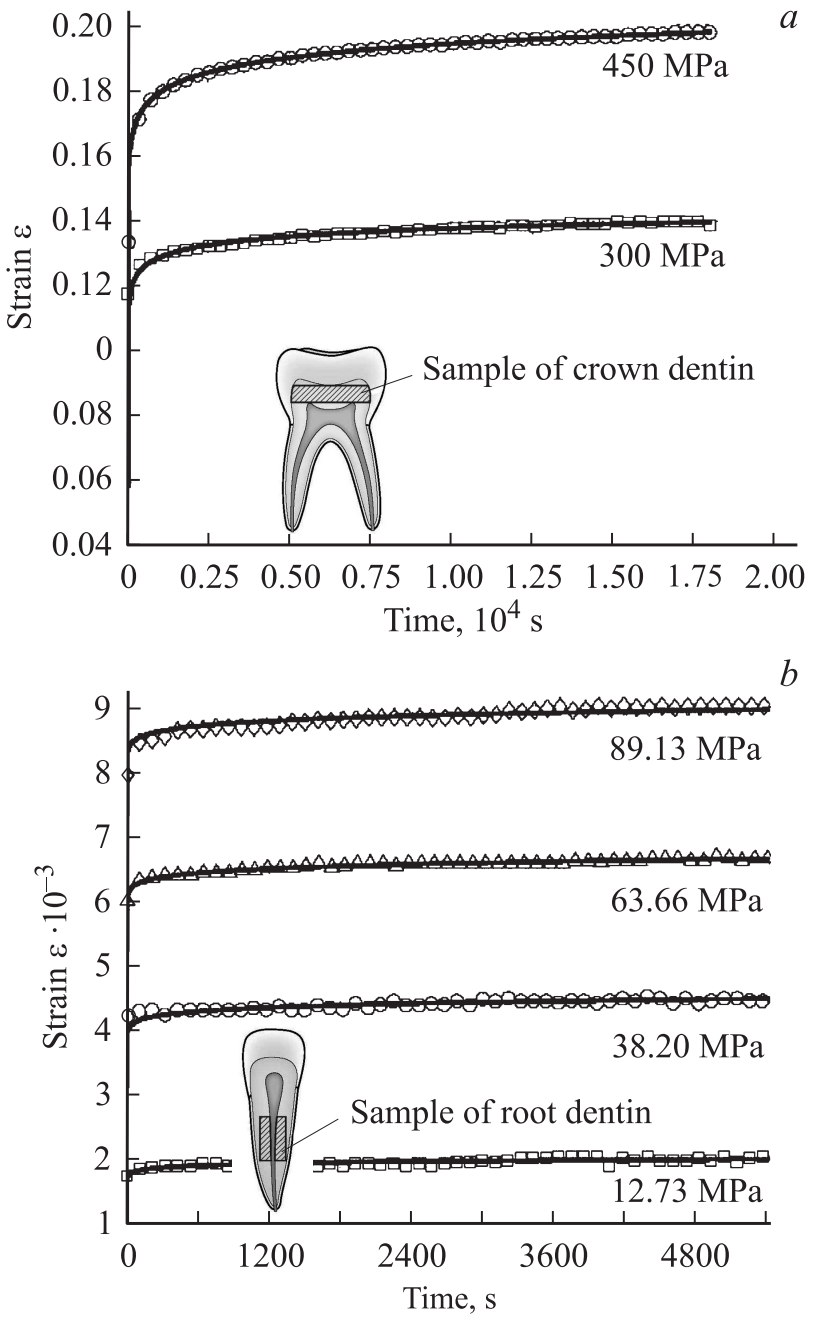

Рис. 2. Экспериментальные (точки) и теоретические (сплошные линии) кривые ползучести дентина при разных уровнях сжимающего напряжения: $a-$ экспериментальные данные из работы [38], $b$ - экспериментальные данные из работы [18]. На вставках показаны схемы вырезки образцов дентина из коронки $(a)$ и из корня $(b)$ зуба.

обоих наборов экспериментальных данных отношение $E_{0} / E_{\infty} \approx 3$. При увеличении приложенного напряжения абсолютная величина параметра $\beta$ падает, что соответствует росту времени релаксации.

Предложенный и опробованный метод представляется достаточно простым и эффективным методом определения вязкоупругих свойств дентина, что открывает новые возможности решения задач механики для этого и подобных ему природных композитных материалов.

\section{3. Физические механизмы пластичности дентина}

Теоретическое описание механического поведения дентина требует понимания физических механизмов пластичности и разрушения на микроуровне. На сегодняш- 

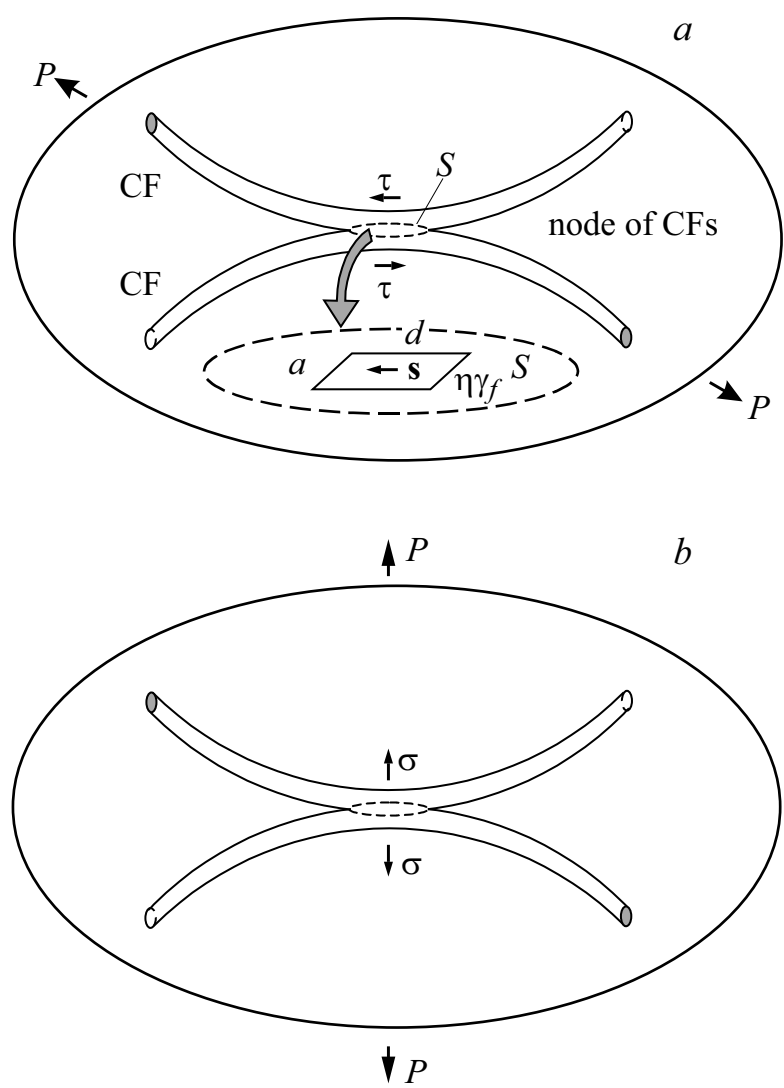

Pис. 3. Схемы нагружения образцов дентина. На площадке контакта двух КВ (CFs) в узле сетки возникает $(a)$ сдвиговое напряжение $\tau$, вызывающее относительный сдвиг КВ, или (b) растягивающее напряжение $\sigma$, приводящее к отделению одного КВ от другого. Здесь $P-$ приложенная нагрузка, $S-$ площадь контакта в узле КВ. На вставке показаны площадка контакта и прямоугольная скользящая петля частичной дислокации с размерами $d \times a$, вектором Бюргерса $\mathbf{s}$ и энергией дефекта упаковки $\eta \gamma_{f}$.

ний день это представляет собой достаточно сложную и нерешенную задачу, отчасти из-за очень малого числа достоверных электронно-микроскопических исследований структуры дентина на микроуровне.

Как уже отмечалось, основным компонентом структуры дентина является сетка переплетенных органических КВ, которые соединены друг с другом множеством узлов. Эта сетка заполняет пространство между ДК. Естественно предположить, что на масштабном уровне такой сетки КВ основными микроскопическими механизмами необратимой (пластической) деформации могут служить: 1) относительный сдвиг КВ в узлах сетки (рис. $3, a), 2$ ) отделение КВ в этих узлах друг от друга (рис. $3, b)$ и 3) продольное необратимое растяжение самих КВ.

Рассмотрим сначала первый из этих микромеханизмов. Следуя работе [39], представим относительный сдвиг $s$ КВ как зарождение дислокационной петли с размерами $d \times a$ в сплошной упругоизотропной среде.
Появление такой петли приводит к изменению полной энергии системы на величину

$$
\Delta W=W_{e l}+W_{c}+W_{\gamma}-A,
$$

где учтены энергии упругого поля дислокационной петли $W_{e l}$, энергия ее ядра $W_{c}$, энергия дефекта упаковки $W_{\gamma}$ и работа $A$, действующего в плоскости петли, сдвигового напряжения $\tau$ по образованию этой петли. Рассмотрим для простоты случай квадратной петли: $d=a$. Тогда для упругой энергии $W_{e l}$ можно записать выражение $[39,40]$

$$
W_{e l} \approx D s^{2} d(2-v)\left(\ln \frac{d}{s}-0.77\right),
$$

где $D=E_{f} /\left[4 \pi\left(1-v^{2}\right)\right], E_{f}-$ эффективный модуль Юнга узла сетки КВ, $v$-эффективный коэффициент Пуассона этого узла, и принято, что радиус экранировки поля петли на ее линии составляет величину порядка $s[39,40]$.

Энергию ядра дислокационной петли можно аппроксимировать обычным выражением [39]

$$
W_{c} \approx D s^{2} d(2-v)
$$

а энергию дефекта упаковки — выражением [41]

$$
W_{\gamma} \approx \eta \gamma_{f}(d-2 s)^{2},
$$

где безразмерный коэффициент $\eta \leq 1$ определяет удельную энергию дефекта упаковки как долю от удельной поверхностной энергии КВ $\gamma_{f}$.

Работа по расширению петли, которую производит приложенное сдвиговое напряжение $\tau$ при пластическом сдвиге на величину $s$, составляет

$$
A=\tau s d^{2} \text {. }
$$

Подстановка выражений (8)-(11) в формулу (7) дает изменение энергии в виде

$$
\begin{gathered}
\Delta W=D s^{2} d\left[(2-v)\left(\ln \frac{d}{s}+0.23\right)\right. \\
\left.+\frac{\eta \gamma_{f}(d-2 s)^{2}}{D s^{2} d}-\frac{\tau d}{D s}\right] .
\end{gathered}
$$

Используя формулу (12) и характерные значения параметров $E_{f} \approx 0.6 \mathrm{GPa}$ [42], $v \approx 0.35$ [43], $\gamma_{f}=0.2 \mathrm{~J} / \mathrm{m}^{2}[44]$ и $\eta \approx 1$, можно численно построить карты изменения энергии $\Delta W$ в координатах $(s, d)$ для разных значений $\tau$. На рис. 4 показаны две такие карты, построенные для $\tau=140$ и $300 \mathrm{MPa}$. Стрелки показывают путь эволюции дислокационной петли: при относительно малых внешних напряжениях на начальных стадиях величина сдвига $s$ растет одновременно с размером петли $d$, но при размерах петли больше нескольких нанометров величина сдвига $s$ продолжает нарастать без существенного увеличения размера $d$, который стремится к значению порядка $7 \mathrm{~nm}$ (рис. 4,a). 

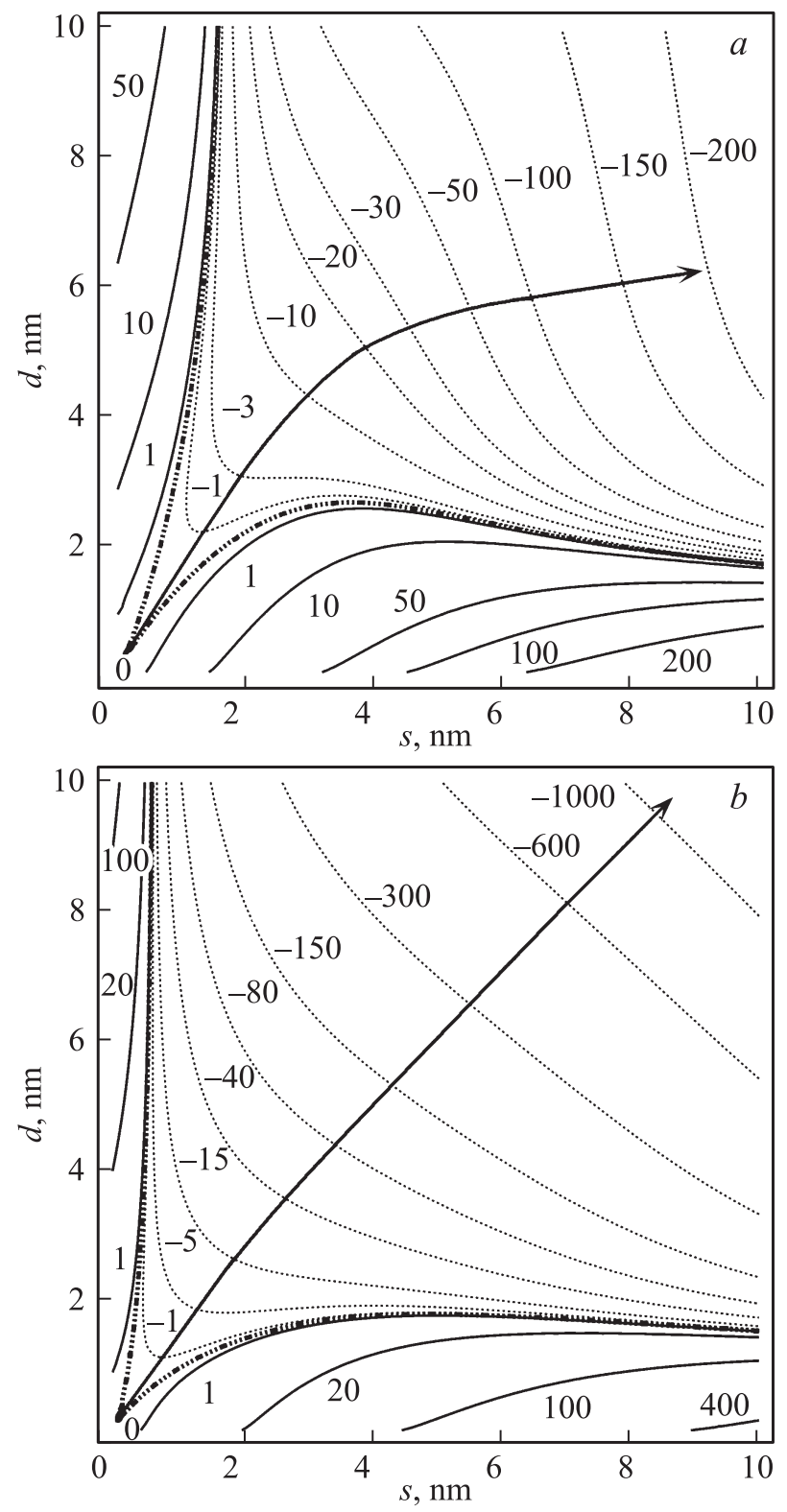

Рис. 4. Карты изменения энергии системы при зарождении дислокационной петли в зависимости от еe размера $d$ и величины сдвига $s$ при $\tau=140 \mathrm{MPa}(a)$ и $300 \mathrm{MPa}(b)$.

При бо́льших внешних напряжениях размер петли $d$ продолжает равномерно расти с увеличением величины сдвига $s$ (рис. 4,b), в пределе экстремально больших напряжений стремясь к постоянному сдвигу при неограниченном росте размера петли $d$.

При достаточно малых значениях $\tau$ изменение энергии $\Delta W$ положительно для любых значений $s$ и $d$ (такие карты здесь не показаны). Это означает, что ни зарождение дислокационных петель, ни проскальзывание КВ в местах их соединения не возможны. Соответственно, пределом текучести для этого случая можно считать напряжение, при котором под действием внешней нагрузки становится возможно гомогенное зарождение дислокационной петли размером порядка нескольких нанометров. Используя карты $\Delta W(s, d)$, подобные представленным на рис. 4, можно найти критическое напряжение, при котором исчезает энергетический барьер для зарождения дислокационной петли и появляются линии с $\Delta W(s, d)<0$ при самых малых $s$ и $d$. Расчеты показывают, что для наблюдаемого в эксперименте разброса механических характеристик дентина это напряжение сдвига составляет $\tau_{s} \approx 120-140 \mathrm{MPa}$. Соответствующее критическое значение приложенного растягивающего напряжения $\sigma_{s}$ для однородного материала определяется как $\sigma_{s}=M \tau_{s}$ при $M \approx 2$. В композитной структуре коллагена фактор $M$ зависит от преимущественной ориентации КВ относительно направления действующей нагрузки и от вида нагружения, но можно предположить, что его значение должно оставаться порядка 2. В этом случае оценочное значение $\sigma_{s} \approx 250-300 \mathrm{MPa} \mathrm{должно} \mathrm{быть}$ близко к критическому напряжению, необходимому для активации этого механизма пластичности в дентине.

Рассмотрим в качестве другого возможного механизма неупругой деформации отрыв друг от друга двух КВ (рис. 3,b), молекулы которых соединены друг с другом ионными и ковалентными связями. Для разрыва двух сцепленных боковыми поверхностями КВ на контактной площадке $S$ требуется некоторое критическое растягивающее напряжение $\sigma_{C}$. Его можно оценить, приравнивая запасенную в области контакта упругую энергию $W_{\sigma}=\sigma^{2} S h /\left(2 E_{f}\right)$ к поверхностной энергии $W_{s}=2 \gamma_{f} S$ [45]. Здесь $h \approx d_{f}-$ линейный размер области контакта (узла КВ). Для минимальных критических напряжений отрыва в случае расположения КВ перпендикулярно направлению растягивающего напряжения имеем

$$
\sigma_{c} \approx 2 \sqrt{\frac{E_{f} \gamma_{f}}{d_{f}}} .
$$

Подставляя сюда характерные значения параметров $E_{f} \approx 0.6 \mathrm{GPa}, \gamma_{f}=0.2 \mathrm{~J} / \mathrm{m}^{2}$ и $d_{f} \approx 200 \mathrm{~nm}$ [46], получаем оценку $\sigma_{c} \approx 34-75 \mathrm{MPa}$. С учетом возможных ориентаций плоскости контакта КВ относительно оси нагружения среднее значение может превышать эту оценку в $1.5-2$ раза.

Наконец, третьим возможным механизмом пластичности дентина может быть продольное растяжение и разрыв отдельных волокон тропоколлагена. Экспериментальные исследования и молекулярно-динамическое компьютерное моделирование разрыва отдельных коллагеновых фибрилл при продольном растяжении были выполнены в работах $[46,47]$. Было показано, что этот разрыв сопровождается относительными сдвигами и локальными разрывами молекул тропоколлагена, которые приводят к пластической деформации, предшествуюшей разрыву КВ в целом [47]. Сообщалось также, что предел текучести отдельных фибрилл зависит от объема КВ: предел текучести уменьшается от 500 до $100 \mathrm{MPa} \mathrm{при}$ увеличении объема КВ до величины порядка $1 \mu \mathrm{m}^{3}$ [47]. 
Испытания на разрыв in vitro показывают более низкие значения предела прочности при растяжении, порядка $230 \pm 160 \mathrm{MPa}$, при деформации до разрушения порядка $80 \pm 44 \%$.

Таким образом, сравнивая между собой характерные значения критических растягивающих напряжений, необходимых для активации трех рассмотренных микромеханизмов пластичности дентина, можно заключить, что наименьшее напряжение (по максимуму - от 70 до $150 \mathrm{MPa}$ ) требуется для отрыва одного КВ от другого в узле сетки КВ. Два других микромеханизма относительный сдвиг КВ в таком узле и необратимое продольное растяжение отдельных КВ - требуют более высоких и примерно одинаковых (в пределах погрешности их определения) критических растягивающих напряжений порядка $200 \mathrm{MPa}$. В то же время разница в этих критических напряжениях не настолько велика, чтобы можно было с уверенностью отбросить вклад двух последних микромеханизмов. Вероятно, следует учитывать возможность одновременного действия всех трех рассмотренных выше микромеханизмов пластичности дентина, отдавая все же определенное предпочтение первому - отрыву КВ друг от друга в узлах сетки КВ.

\section{4. Пластические зоны в вершине трещины отрыва в дентине}

Используя результаты предыдущего раздела, можно оценить размеры и форму пластической зоны в вершине трещины в дентине и сравнить их с экспериментальными изображениями, полученными с помощью просвечивающей электронной микроскопии (см., например, [48]).

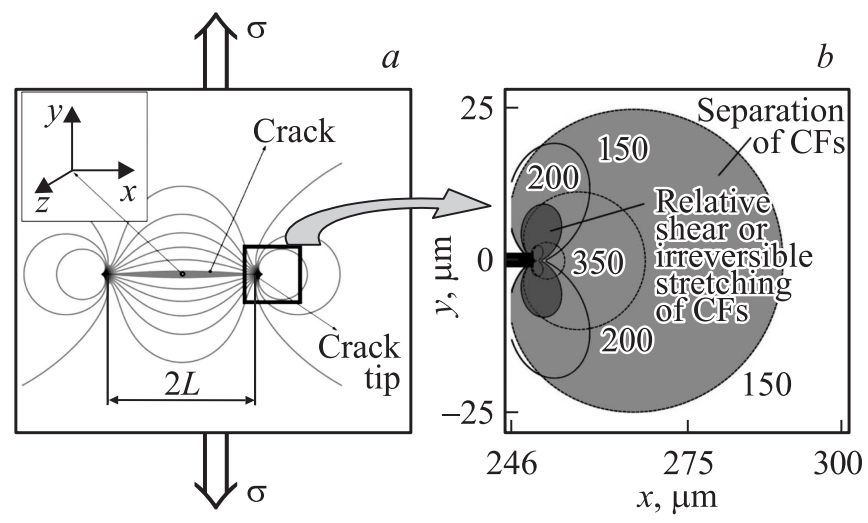

Рис. 5. Пластические зоны вблизи вершины трещины отрыва в дентине для случая $\sigma=50 \mathrm{MPa}$ и $2 L=500 \mu \mathrm{m}: a-$ общий вид нагружаемого образца с трещиной отрыва; $b$ - часть карты гидростатической компоненты тензора напряжений (штриховые линии) и мизесовского сдвигового напряжения (сплошные линии) вблизи вершины трещины; закрашенные области соответствуют пластическим зонам, внутри которых возможна реализация трех механизмов пластичности в дентине - относительного сдвига КВ в месте их соединения или необратимого их удлинения (более темная зона) и отрыва КВ друг от друга в месте их соединения (более светлая зона).
Эксперименты показывают [23], что в случае одноосного сжатия образца трещины могут соответствовать всем трем типам разрушения и расти как вдоль растягивающей нагрузки (в центре образца при сжатии), так и перпендикулярно ей или под углом $45^{\circ}$ к оси нагружения. Для получения конфигурации упругого поля вблизи вершины трещины простого отрыва (первый тип разрушения) воспользуемся ее дислокационной моделью [41]. В этой модели разрыв моделируется непрерывным распределением краевых дислокаций с бесконечно малыми векторами Бюргерса.

Рассмотрим, например, поле напряжений в окрестности трещины длиной $2 L$ (рис. $5, a)$, которая раскрывается в плоскости $x z$ декартовой системы координат $(x, y, z)$ под действием приложенного вдалеке растягивающего напряжения $\sigma$. Ненулевые компоненты этого поля можно представить в компактном виде [49]

$$
\begin{aligned}
\sigma_{x x}= & \frac{\sigma}{2 \sqrt{2} P^{6}}\left\{|x| Q_{+}\left(3 x_{-}^{4}+2 x_{+}^{2} y^{2}-y^{4}-P^{2} r_{+}^{2}\right)\right. \\
& \left.+|y| Q_{-}\left(5 x_{-}^{4}+6 x_{+}^{2} y^{2}+y^{4}+P^{2} r_{+}^{2}\right)\right\}, \\
\sigma_{y y}= & \frac{\sigma}{2 \sqrt{2} P^{6}}\left\{|x| Q_{+}\left(x_{-}^{4}+6 x_{+}^{2} y^{2}+5 y^{4}+P^{2} r_{+}^{2}\right)\right. \\
& \left.-|y| Q_{-}\left(x_{-}^{4}-2 x_{+}^{2} y^{2}-y^{4}+P^{2} r_{+}^{2}\right)\right\}, \\
\sigma_{x y}= & \frac{\sigma \operatorname{sgn}(x y)}{2 \sqrt{2} P^{6}}\left\{|x| Q_{-}\left(x_{-}^{4}-2 x_{+}^{2} y^{2}-3 y^{4}+P^{2} r_{+}^{2}\right)\right. \\
& \left.-|y| Q_{+}\left(3 x_{-}^{4}+2 x_{+}^{2} y^{2}-y^{4}-P^{2} r_{+}^{2}\right)\right\},
\end{aligned}
$$

где

$$
\begin{gathered}
P^{2}=\left[4 x^{2}(y-L)^{2}+r_{-}^{4}\right]^{1 / 2}, \quad Q_{ \pm}=\left(P^{2} \pm r_{-}^{2}\right)^{1 / 2}, \\
x_{ \pm}^{2}=x^{2} \pm L^{2}, \quad y_{ \pm}^{2}=y^{2} \pm L^{2}, \quad r_{ \pm}^{2}=x^{2} \pm y_{ \pm}^{2} .
\end{gathered}
$$

На рис. 5, $b$ показаны карты распределения полей гидростатической компоненты тензора напряжений $\sigma_{m}=\left(\sigma_{x x}+\sigma_{y y}+\sigma_{z z}\right) / 3$, где $\sigma_{z z}=v\left(\sigma_{x x}+\sigma_{y y}\right)$ в нашем случае плоско-деформированного состояния, и сдвигового мизесовского напряжения $\sigma_{y}=\left[\left(\sigma_{x x}-\sigma_{y y}\right)^{2}+\right.$ $\left.+\left(\sigma_{x x}-\sigma_{z z}\right)^{2}+\left(\sigma_{y y}-\sigma_{z z}\right)^{2}+6 \sigma_{x y}^{2}\right]^{1 / 2} / \sqrt{2}$ в окрестности вершины трещины разрыва для случая малого приложенного напряжения $\sigma=50 \mathrm{MPa} \mathrm{при} 2 L=500 \mu \mathrm{m}$. В более светлой части закрашенной области, выделенной пунктирными изолиниями, гидростатическая компонента тензора напряжений превышает $150 \mathrm{MPa}$, и может сработать механизм отрыва КВ друг от друга при их боковом соединении в узле сетки КВ. В более темной части закрашенной области, примыкающей к вершине трещины, мизесовское сдвиговое напряжение, изолинии которого даны сплошными жирными линиями, превышает $200 \mathrm{MPa}$ - напряжение, необходимое для проскальзывания КВ в таких узлах друг по другу или для необратимого растяжения отдельных КВ. Эти об- 
ласти могут рассматриваться как пластические зоны, в которых возможно срабатывание трех описанных выше механизмов пластичности в дентине.

Когда внешнее напряжение становится еще меньше, порядка $10 \mathrm{MPa}$, размер пластической зоны $r^{*}$ составляет несколько сотен нанометров. При внешних напряжениях, превышающих $50 \mathrm{MPa}$, он увеличивается до десятков микрометров (рис. 5), что качественно соответствует имеющимся экспериментальным наблюдениям [33]. При дальнейшем росте внешних напряжений до значений, близких к пределу текучести, порядка 100-150 MРа, пластическая зона вырастает до сотни микрометров. Во всех этих случаях общая конфигурация пластической зоны остается одной и той же.

Заметим, что наша оценка размера пластической зоны $r^{*}$ получена в первом приближении и может быть справедлива в самом начале процесса пластической деформации вблизи вершины трещины в дентине. По мере развития пластической деформации в этой области происходит перераспределение напряжений, и реальный размер пластической зоны $r_{y}$ должен вырасти примерно в два раза: $r_{y} \approx 2 r^{*}$ [50]. Используя результаты экспериментальных измерений коэффициента интенсивности напряжений в вершине трещины $K_{I}$ [31], можно проверить наши оценки для $r_{y}$. Взяв известную формулу $r_{y}=K_{I}^{2} /\left(2 \pi \sigma_{y}^{2}\right)$ и подставив в нее $\sigma_{y} \approx 200 \mathrm{MPa}$ и $K_{I}=K_{I c} \approx 2.5 \mathrm{MPa} \cdot \mathrm{m}^{1 / 2}$ [31], получаем $r_{y} \approx 150 \mu \mathrm{m}$. Это значение соответствует оценкам размера зоны предразрушения в окрестности трещины, которая определялась по разрывам минерализованных оболочек ДК [30]. Для меньших напряжений и, следовательно, меньших значений коэффициента интенсивности напряжений размер пластической зоны можно оценить в десятки микрометров, что соответствует результатам нашей модели.

\section{5. Заключение}

В заключение суммируем полученные выше результаты.

1. Предложена теоретическая модель, описывающая эффективные вязкоупругие свойств дентина при одноосном нагружении в рамках теории линейной вязкоупругости Работнова с использованием дробно-экспоненциальных функций в качестве ядер ползучести и релаксации. Из сравнения теоретических кривых ползучести с данными экспериментов по статическому сжатию образцов дентина получены численные значения мгновенного и долговременного модулей Юнга, а также других характеристик вязкоупругости дентина. Показано, что предложенный и опробованный метод достаточно прост и эффективен для определения вязкоупругих свойств дентина.

2. Рассмотрены теоретические модели механизмов пластической деформации дентина на микроскопическом уровне: 1) взаимное проскальзывание КВ в месте контакта их боковых поверхностей в узлах сетки КВ, 2) отрыв этих КВ друг от друга и 3) необратимое удлинение отдельных КВ. Сделаны численные оценки уровней напряжений, которые необходимы для срабатывания этих механизмов. Показано, что второй механизм (отрыв КВ друг от друга) требует меньших напряжений и может считаться более предпочтительным, чем два других.

3. Исследованы размеры и конфигурации пластических зон в вершине трещины простого отрыва, отвечающих действию рассмотренных механизмов пластичности дентина. Показано, что пластическая зона, обусловленная действием первого и третьего механизмов (взаимное проскальзывание КВ в узле сетки КВ и необратимое удлинение отдельных КВ), сильнее локализована вблизи вершины трещины, чем пластическая зона, обусловленная действием второго механизма (отрыв КВ друг от друга). Сделаны оценки протяженности этих пластических зон в зависимости от величины приложенного напряжения. Показано, что когда это напряжение мало (порядка $10 \mathrm{MPa}$ ), размер большей пластической зоны составляет несколько сотен нанометров, при большем напряжении (порядка $50 \mathrm{MPa}$ ), он увеличивается до десятков микрометров, и при напряжении, близком к пределу текучести (порядка 100-150 MPa), он вырастает до сотни микрометров.

\section{Список литературы}

[1] С.Ю. Бывальцева, 3.В. Доржиева. Строение твердых тканей зуба. ИГМУ, Иркутск. (2013). 36 с.

[2] А.В. Беликов, В.Н. Грисимов, А.В. Скрипник, К.В. Шатилова. Лазеры в стоматологии. Ун-т ИТМО, СПб. (2015). Ч. $1.108 \mathrm{c}$.

[3] M.A. Meyers, P.Y. Chen. Biological Material Science. Cambridge. (2014).

[4] R. Elbaum, E. Tal, A.I. Perets, D. Oron, D. Ziskind, Y. Silberberg, H.D. Wagner. J. Dentistry 35, 150 (2007).

[5] D. Zaytsev, A. Ivashov, P. Panfilov. Mater. Lett. 138, 219 (2015).

[6] Z. Qin, A. Gautieri, A.K. Nair, H. Inbar, M.J. Buehler. Langmuir 28, 1982 (2012).

[7] Y. Liu, R. Ballarini, S.J. Eppell. Interface Focus 6, 20150080 (2016).

[8] A. Gautieri, M.J. Buehler, A. Redaelli. J. Mech. Behav. Biomed. Mater. 2, 130 (2009).

[9] G.W. Marshall jr, S.J. Marshall, J.H. Kinney, M. Balooch. J. Dentistry 25, 441 (1997).

[10] J.H. Kinney, S.J. Marshall, G.W. Marshall. Crit. Rev. Oral Biol. Med. 14, 13 (2003).

[11] Y.R. Zhang, W. Du, X.D. Zhou, H.Y. Yu, Intern. J. Oral Sci. 6, 61 (2014).

[12] G.V. Black. Dental Cosmos 37, 353, 469, 553, 637, 737 (1895).

[13] http:/quod.lib.umich.edu/d/dencos/acf8385.0037.001/ $1: 203$ ?view $=$ toc

[14] F. Peyton, D.B. Mahler, B. Hershenov. J. Dental Res. 31, 366 (1952). 
[15] J.W. Stanford, G.C. Paffenbarger, J.W. Kumpula, W.T. Sweeney. J. Am. Dental Association 57, 487 (1958).

[16] M. Balooch, I.C. Wu-Magidi, A. Balazs, A.S. Lundkvist, S.J. Marshall, G.W. Marshall, W.J. Siekhaus, J.H. Kinney. J. Biomed. Mater. Res. 40, 539 (1998).

[17] B. Huo, Q.S. Zheng, Q. Zhang, J.D. Wang. Acta Mech. Sin. 16, 75 (2000).

[18] J. Jantarat, J.E.A. Palamara, C. Linder, H.H. Messer. Dental Mater. 18, 486 (2002).

[19] D.H. Pashley, K.A. Agee, J.C. Wataha, F. Rueggeberg, L. Ceballos, K. Itou, M. Yoshiyama, R.M. Carvalho, F.R. Tay. Dental Mater. 19, 700 (2003).

[20] M.G. Duncanson, E. Korostoff. J. Dental Res. 54, 1207 (1975).

[21] D. Zaytsev, S. Grigoriev, P. Panfilov. Int. J. Biomater. 2012, 854539 (2012).

[22] D. Zaytsev, P. Panfilov. Mater. Sci. Eng. C 45, 205 (2014).

[23] D. Zaytsev. Mater. Sci. Eng. C 49, 101 (2015).

[24] Д.В. Зайцев. Физические механизмы деформации и разрушения в материалах с развитой иерархической структурой. Дентин и эмаль зубов. Автореф. докт. дис. Екатеринбург (2015). $48 \mathrm{c}$.

[25] T. Jafarzadeh, M. Erfan, D.C. Watts. J. Dentistry Tehran Univer. Med. Sci. 1, 5 (2014).

[26] R. Halgaš, J. Dusza, J. Kaiferová, L. Kovácsová, N. Markovská. Ceramics-Silikáty 57, 92 (2013).

[27] L.M. Petrovic, D.T. Spasic, T.M. Atanackovic. Dental Mater. 21, 125 (2008).

[28] Y.H. Cui, X. Wang, Y.X. Zhang, F.J. He. Philos. Mag. 90, 1197 (2010).

[29] S.F. Chuang, S.Y. Lin, P.J. Wei, C.F. Han, J.F. Lin, H.C. Chang. J. Biomech. 48, 2155 (2015).

[30] R.K. Nalla, J.H. Kinney, R.O. Ritchie. J. Biomed. Mater. Res. A 67, 484 (2003).

[31] R.K. Nalla, J.H. Kinney, R.O. Ritchie. Biomater. 24, 3955 (2003).

[32] K.J. Koester, J.W. Ager III, R.O. Ritchie. Biomater. 29, 1318 (2008).

[33] D. Zaytsev, A. Ivashov, J.V. Mandra, P. Panfilov. Mater. Sci. Eng. C 41, 83 (2014).

[34] P. Panfilov, A. Kabanova, J. Guo, Z. Zhang. Mater. Sci. Eng. C 71, 994 (2017).

[35] Ю.Н. Работнов. Прикладная математика и механика 12, 1 , 53 (1948).

[36] G.W. Scott Blair, F.M.V. Coppen. Proc. Roy. Soc. B 128, 109 (1939).

[37] G.W. Scott Blair, F.M.V. Coppen. Am. J. Psychol. 56, 234 (1943).

[38] S. Seyedkavoosi, D. Zaytsev, B. Drach, P. Panfilov, M.Yu. Gutkin, I. Sevostianov. Int. J. Eng. Sci. 111, 52 (2017).

[39] М.Ю. Гуткин, И.А. Овидько. ФТТ 50, 630 (2008).

[40] M.Yu. Gutkin, A.G. Sheinerman. Phys. Status Solidi B 241, 1810 (2004).

[41] Дж. Хирт, Й. Лоте. Теория дислокаций. Атомиздат, М. (1972), $600 \mathrm{c}$.

[42] A. Barkaoui, A. Bettamer, R. Hambli. Procedia Eng. 10, 3185 (2011).

[43] F. Hang, H.S. Gupta, A.H. Barber. J. R. Soc. Interface 11, 20130993 (2014).
[44] X. Wang, T. Wu, W. Wanga, C. Huanga, X. Jin. Mater. Sci. Eng. C 58, 750 (2016).

[45] В.Л. Попов. Механика контактного взаимодействия и физика трения. От нанотрибологии до механики землетрясений. Физматлит, М. (2013). 352 с.

[46] M.J. Buehler. J. Mech. Behav. Biomed. Mater. 1, 59 (2008).

[47] Z.L. Shen, M.R. Dodge, H. Kahn, R. Ballarini, S.J. Eppell. Biophys. J. 95, 3956 (2008).

[48] E.N. Borodin, M.Yu. Gutkin, K.N. Mikaelyan, P. Panfilov. Scripta Mater. 133, 45 (2017).

[49] M.Yu. Gutkin, I.A. Ovid'ko, N.V. Skiba. Philos. Mag. 88, 1137 (2008).

[50] A.F. Liu. Mechanics and Mechanisms of Fracture: An Introduction, ASM International, Materials Park, Ohio, USA. (2005). 